\title{
Limited visual control of the intelligibility of speech in face-to-face dialogue
}

\author{
A. H. ANDERSON \\ University of Glasgow, Glasgow, Scotland \\ E. G. BARD and C. SOTILLO \\ University of Edinburgh, Edinburgh, Scotland \\ and \\ A. NEWLANDS and G. DOHERTY-SNEDDON \\ University of Glasgow, Glasgow, Scotland
}

\begin{abstract}
Speakers are thought to articulate individual words in running speech less carefully whenever additional nonacoustic information can help listeners recognize what is said (Fowler \& Housum, 1987; Lieberman, 1963). Comparing single words excerpted from spontaneous dialogues and control tokens of the same words read by the same speakers in lists, Experiment 1 yielded a significant but general effect of visual context: Tokens introducing 71 new entities in dialogues in which participants could see one another's faces were more degraded (less intelligible to 54 naive listeners) than were tokens of the same words from dialogues with sight lines blocked. Loss of clarity was not keyed to momentto-moment visual behavior. Subjects with clear sight lines looked at each other too rarely to account for the observed effect. Experiment 2 revealed that tokens of 60 words uttered while subjects were looking at each other were significantly less degraded (in length and in intelligibility to 72 subjects) vis-à-vis controls than were spontaneous tokens of the same words produced when subjects were looking elsewhere. Intelligibility loss was mitigated only when listeners looked at speakers. Two separate visual effects are discussed, one of the global availability and the other of the local use of the interlocutor's face.
\end{abstract}

No two tokens of the same word form are ever fully identical, even when they are uttered within seconds of each other by the same speaker. Explanations for these differences lie at every level of linguistic description. Although most spoken words are fully recognizable within their linguistic contexts, some differences in pronunciation affect the intelligibility of a word when it is excerpted from that context and presented as an isolated acoustic form. It has been argued that many such differences in pronunciation are based on the speaker's view of the listener's needs (Bolinger, 1963, 1981; Chafe, 1974; Lieberman, 1963; Lindblom, 1990). This paper deals with how precise that view is, and, in particular, how closely speakers' performance reflects listeners' access to information that can be obtained from the speaker's face (Massaro \& Cohen, 1983).

This work was supported by the ESRC(UK)-funded Human Communication Research Centre and by ESRC Project Grant R000233560 to A.H.A., C. O'Malley, and V. Bruce. A preliminary version of this paper was presented at the summer 1994 meeting of the Experimental Psychology Society. The authors are grateful to Simon Garrod, Bruno Repp, Quentin Summerfield, Dominic Massaro, and Joanne Miller for comments on the manuscript. The remaining errors are the authors'. G. Doherty-Sneddon is now at the Department of Psychology, University of Stirling. Correspondence should be addiessed to A. H. Anderson, HCRC, Department of Psychology, University of Glasgow, 56 Hillhead Street, Glasgow G12 9YR, Scotland (e-mail: anne@psy. gla.ac.uk).
There is ample evidence that speakers adjust their pronunciation of words in running speech to complement other information that might help their listeners recognize those words, particularly information that can be gleaned from the remainder of the discourse and of its context. For example, the clearest tokens of words are those read from lists in which there are no contextual cues to the words' identities: Excerpted from their phonetic contexts and played to naive subjects, these "citation forms" may be recognized without difficulty. In contrast, word tokens extracted from running speech are harder to identify correctly. Pollack and Pickett (1963) reported an average of $50 \%$ correct identification for single words, though intelligibility scores ranged from $0 \%$ to $100 \%$. The particular changes in pronunciation that affect intelligibility are thought to include fairly well understood phenomena like increased speed, phonological reduction, and phonological assimilation both within and across word boundaries. The processes that create these changes may be described as casual or careless articulation. The question we address here is why these processes apply.

\section{WHY DO SPEAKERS VARY CARE IN ARTICULATION?}

Lieberman (1963) postulated that speakers are sensitive to the distribution of information used in recognizing 
spoken words. He showed that the more predictable a word is from its sentence context-that is, the more information about the word's identity is held in the rest of its sentence-the less clear the word token will be. Lieberman's observation was based on pairs of word tokens extracted from readings of sentence contexts like the following:

1. The word you are about to hear is nine.

\section{A stitch in time saves nine.}

For the shared word, like nine, the token taken from the utterance in which it was more predictable in a cloze procedure-that is, (2) was both shorter and less intelligible than the corresponding, but less redundant, item in (1). In general, the higher the predictability of a word, the lower its intelligibility when isolated (Bard \& Anderson, 1983; Fowler \& Housum, 1987; Hunnicutt, 1985).

Other sources for pertinent information are distributed throughout the discourse in which words are uttered and beyond. The token of a word that introduces a New entity into a discourse is typically longer and clearer when heard in isolation than is a token that refers to the entity when it is already Given by virtue of having been mentioned before. The effect is not an outcome of the mechanical process of uttering a word twice. It is not found in word lists but only in connected meaningful discourse and is most marked when speakers communicate with a listener (Fowler, 1988). It is absent when repetitions do not refer to the same object or event (Bard, Lowe, \& Altmann, 1989), but it is found when the two tokens are coreferential (Bard et al., 1989; Fowler \& Housum, 1987). Moreover, the effect does not require repetition. Entities that are physically present when mentioned (what Prince, 1981 , called "situationally evoked") are also Given. Word tokens that make initial reference to situationally Given entities are said less clearly than tokens whose referents are absent (Bard \& Anderson, 1994).

A parallel sensitivity to discourse and physical context affects the form of referring expressions and the nature of descriptions: Like word duration or clarity, referring expressions tend to be reduced when speakers share more relevant information with their listeners (Isaacs \& Clark, 1987; Schober \& Clark, 1989; Wilkes-Gibbs \& Clark, 1992). Ariel (1990) has shown that shorter and syntactically simpler referring expressions are used when the referent is more accessible - that is, when listeners or readers can be assumed to have more or more salient information about it. Though much of Ariel's discussion relates to the syntax of referring expressions, she also distinguished stressed, unstressed, "cliticized," and null tokens of pronouns - forms ordered by decreasing phonological substance and increasing accessibility of referents. In fact, changes in the pronunciation of words have often been described in similar terms. The pronunciations that carry unexpected information (Bolinger, 1963, 1981) or distinguish New from Given (Chafe, 1974; Hawkins \& Warren, 1994; Terken \& Nooteboom, 1987) also pair the more elaborate forms with the less accessible referents.
Because the adjustment seems to be made in response to what the listener might know, modifications in the clarity of speech appear to offer an elegant extension of the Gricean principle of cooperation (Grice, 1975) if we translate the maxim of quantity to the articulatory domain: We articulate with enough care to maintain communication (see Fowler \& Levy, 1991; Koopmans-van Beinum, 1980 ) or, as Lindblom (1990) has put it, we hyper- or hypoarticulate as the listener's situation requires.

Like all the Gricean relationships, however, this one demands extraordinary powers on the part of the speaker if it is to work properly. For example, it would not be particularly cooperative for a speaker to treat an item that he or she can see as Given if the listener cannot see it, too, or to articulate a word carelessly when it is wholly predictable in context to the speaker but not to the listener. To maintain any cooperative characteristics, the speaker must develop and update a model of the listener's knowledge and current needs. The literature on dialogue indicates that an accurate model may often be hard to maintain: Apparently shared views need frequent realignment (WilkesGibbs \& Clark, 1992). For a view of the listener's understanding that was always correct, the speaker would have, in effect, to read the listener's mind.

Unsurprisingly, no one has yet shown that speakers constantly update their appreciation of listeners' knowledge. In fact, there is reason to believe that speakers choose a simple and often adequate solution to the problem created by Gricean relationships: They act as if their own view of predictability or Givenness were an adequate model for their listeners'. When speakers and listeners share a physical location, a certain amount of recent experience, and general cognitive abilities, the simple solution should work nicely. When any of these is lacking, mistakes will occur. We have evidence of two. Bard and Anderson (1983) showed that parents adjusted the pronunciation of words to predictability from the surrounding sentence to the same degree, whether the listener was a very young child (18 months to 3 years) or an adult. The fact that a child of this age was unlikely to have mastered enough syntax to be able to profit fully from sentential context seemed to have no effect. Similarly Bard and Anderson (1994) replicated Fowler and Housum's (1987) repetition effect in parental speech to small children (see also Fisher \& Tokura, 1995). Parents articulated less carefully despite the fact that they were repeating themselves precisely because the child had not noticed the first mention of the item. Yet as far as anyone could tell by observing the listeners, the Given item was New for them, however over-Given it was for the speakers. Achieving good insight into the appreciations of a small child may be difficult, but no such excuse applies to a distinctly un-Gricean effect in adult-adult speech. Office workers using a dictating machine to create documents degraded second tokens of words within seconds of intentionally erasing the corresponding first mentions so that the degraded tokens in the revised message would be the first 
the typist would hear (Bard et al., 1989). Again, speakers responded to the information that only they could use.

\section{LOOKING FOR FINE CONTROL}

Such examples suggest that speakers do not maintain an accurate model of the information that is really available to their listeners. While composing and comprehending messages, they may simply not have the cognitive resources to track listeners' appreciation of the discourse and its surroundings, even insofar as normal powers of perception would allow them to do so. This proposal is conjecture on our part: It is based on speakers' failure to reflect in production certain factors that should have been taken into account if they were revising their models of the listener moment by moment. The present paper directly tests the hypothesis that speakers' models of their listeners' needs are more likely to be a reflection of their own knowledge than a frequently updated model of their listeners'.

To obtain an independent indication of the speaker's attention, we turn to the visual domain. First, by manipulating what speakers and listeners can see, we can determine whether speakers adjust their speech when pertinent visual information is available. Then, by observing where speakers and listeners actually look, we can discover whether listeners are using visual cues to word identity and whether speakers are actually keying adjustments in their speech to observations of the listener's behavior.

The visual information of interest here is provided by the speakers' faces. In natural conversation, the face presents at least two kinds of information--what we might call interactive information and information about articulation itself. We will discuss the information carried by each in the next two sections. Here we need to consider how appropriate it is to propose that articulatory detail might be adjusted to such cues.

We assume that there is no great problem in proposing that visual information per se can be integrated with auditory linguistic information. Although most of the effects on care in pronunciation are linguistic, we have already shown that pertinent information can be conveyed visually: The physical presence of the referent affects the pronunciation of first mentions. Such interactive visual cues as facial expressions, paralinguistic gestures, and indications of comprehension or confusion can also be thought of as pertinent context - something outside the acoustic signal that can be recruited to help decode it. Visually conveyed articulatory information would, however, seem to be a less obvious source of pronunciation effects, because it appears to be an inalienable part of the act of articulation, rather than an independent but concurrent information source.

It is important to keep in mind, however, that the issue is not the mechanics of speech production but the transmission of cues to what is produced. Visual cues to articulation are dissociable from auditory cues both in production and in perception. When auditory information is attenuated in whispering, lip movements need not be attenuated. In fact, speakers may exaggerate lip movements in noisy conditions when they cannot expect to be heard or when being audible is undesirable. Audible speech without visible correlates is found even more widely, and not just in ventriloquists' specialist performances. In telephone conversations, radio broadcasts, audio recordings, in fact in any linguistic interaction not carried on face to face, visual cues to articulation are simply unavailable.

As long as speakers can tell which aspects of their output are transmitted and decide how information will be distributed between sound and sight, it should be possible for them to make the appropriate adjustments. Both these conditions are generally met. Any normal speaker above the age of 5 or so is likely to know whether he/she can be seen, heard, or both. In mouthing when no audience will hear them, speakers show the required awareness and the corresponding control of transmission modalities. We would predict that auditory cues to word identity should be relatively enhanced when speakers cannot be seen, and relatively degraded when they can be. What that enhancement would do to visual cues is irrelevant if the visual cues are not transmitted. What degrading articulation would do to concomitant transmitted visual cues is not clear, but even very careless articulation is unlikely to destroy visual cues altogether.

We move on in the next two sections to consider the particular visual cues that interlocutors' faces providefirst the interactive cues and then the articulatory. We then consider the possibility that speakers and listeners monitor for these cues regularly and frequently. Finally, we present experiments that test how sensitively and in what ways speakers key auditory clarity to visual information.

\section{Interactive Facial Cues}

In face-to-face conversation, speakers and listeners use such visual cues as facial expressions and glances to augment purely linguistic communication. They may modulate the meaning conveyed by speech or convey meaning themselves.

Facial expressions are cues to how a listener is reacting to a message or situation. In some circumstances these have considerably more impact than the verbal contents of comments or the tone of voice on an observer's judgment of a speaker's reaction to a previous message (Mehribian, 1972; O'Sullivan, Ekman, Friesen, \& Scherer, 1985; Walker, 1977).

Glances or gaze, like utterances, are exchanged in faceto-face conversation. Gaze has been described as playing a role in managing turn taking (Duncan \& Fiske, 1977; Kendon, 1967; but see Beattie, 1983). Gaze can also be used to indicate that an interlocutor is attentive to a message. On the basis of an analysis of $50 \mathrm{~h}$ of natural conversations, Goodwin (1981) concluded that speakers want others to show that they are listening and so look up to elicit responsive gazes. Gaze also has a paralinguistic function that modulates prosodic effects: Walker and Trimboli 
(1983) claim that brief glances or "eye flashes" help speakers emphasize particular words or phrases.

Moments of eye contact may even fulfill the role that whole utterances would otherwise carry. Face-to-face dialogues are differently structured from comparable dialogues in which visual cues are not available. For this reason, route communication dialogues have been found to be significantly shorter if conducted face to face (Boyle, Anderson, \& Newlands, 1994). Using the corpus from which we will draw materials for the present work, Boyle et al. showed that although participants communicated a route with equal success in the two conditions, the face-to-face dialogues contained significantly less speech. The incidence of gaze from the subjects trying to follow directions was significantly higher when communicative difficulties arose-for example, when their own maps did not show the landmark they were supposed to be negotiating. Doherty-Sneddon et al. (in press) further showed that at those junctures where participants most often looked at one another while describing something, subjects who could not see their partners had to resort to additional verbal checks on the partner's understanding. Clearly, visually transmitted information functioned as a contribution to the dialogue.

Since the interactive functions of the face modulate and add to dialogue, they should constitute part of the discourse context in which participants speak. Like the rest of that context, they should be able to affect the predictability of a word and consequently the way in which it is articulated.

\section{Facial Cues to Articulation}

Although interactive facial cues are intermittent, facial cues to articulation are more or less continuous. The term lip shape can be used, perhaps confusingly, to cover externally visible indications of articulatory movements. Some visual cues to articulation are actually in the form of phonetically pertinent lip shapes, but in general the information is dynamic and includes jaw movements, tongue protrusions, and the like. These display the articulatory characteristics of classes of speech sounds- the rounding of vowels and the place of articulation of some consonants, in particular-and support discrimination for something like 16 of the 40 phonemes of English (Erber, 1974). The relationship between visual and auditory cues varies. For consonants, visual and auditory cues are complementary: Visual cues are most powerful in making those phonetic distinctions that are most difficult to make on the basis of auditory information alone (Walden, Prosek, \& Worthington, 1975); for vowels, visual and auditory cues are redundant, supporting the same distinctions.

The utility of lip shape has been convincingly demonstrated in laboratory conditions. Visual cues can help listeners to identify stimuli as diverse as isolated syllables (McGurk \& MacDonald, 1976) and words in continuous prose in the listener's first or second language (Reisberg, McLean, \& Goldfield, 1987). Visual information proves useful when sound is a deficient clue to what was said (Miller, Heise, \& Lichten, 1951; Reisberg et al., 1987;
Sumby \& Pollack, 1954; Summerfield, 1987) and when the listener has a hearing impairment (Walden et al., 1975).

Visual cues seem to be used within the overall perceptual system that applies auditory cues to the identification of spoken words. Both kinds of sensory information appear to interact with higher level linguistic regularities in recognition tasks. Like audio-only recordings, silent visual presentations of the speaker's face yield better recognition for materials with more linguistic structure: Normally hearing subjects' recognition rises from nonsense syllables $(27 \%)$, through isolated words, to words in stories or short phrases (72\%; Gailey, 1987). Massaro (1989) has gone so far as to suggest that whatever commands our attention while we listen to speech, lip shape cues are so compelling that we will always observe them to some extent.

Certainly, the uptake of visual clues in speech perception is no ad hoc strategy, but is sufficiently related to the use of auditory cues to yield the set of striking illusions called the McGurk effect (McGurk \& MacDonald, 1976). When visual and auditory clues to place of articulation disagree, the auditory percept integrates the two, often corresponding to a place intermediate between visually and aurally indicated sites.

In all this work, however, subjects have been asked only to identify what was said. There was no need to interact with the apparent speaker. In conversation, deaf people certainly do make use of visual cues, but it is less certain to what extent unimpaired speaker-listeners use this rich source of articulatory information. In fact, several lines of research in social psychology indicate that the occasions on which interlocutors gaze at each other are severely limited.

\section{On Not Gazing at Faces}

Even in conditions in which there are few visual distractions, Argyle (1990) has reported, gaze levels by any one speaker rarely rise above $50 \%$ of total time in conversation and are often considerably lower (Abele, 1986; Exline, 1972; Kendon, 1967; Nielson, 1962). Estimates of mutual gaze/eye contact between speaker and listener are lower still, at about $22 \%$ in data samples provided by Kendon. Three reasons are offered for these low figures.

First, eye contact is said to indicate intimacy, and intimacy, it is claimed, must be negotiated to a suitable equilibrium in each conversation (Argyle \& Dean, 1965). In evidence, Argyle (1990) has shown that pairs increasing intimacy by sitting closer together compensate by looking at each other less. Assiduously tracking lip shape or constantly checking for the listener's confused expressions might simply be "forward" behavior.

Second, looking at a partner's face may distract the speaker from thinking and planning speech. Speakers look away from their partner more than listeners do (Argyle \& Ingham, 1972; Exline, Jones, \& Maciorowski, 1977; Kendon, 1967). Speakers are most likely to look away either at the beginning of a conversational turn, when they may be planning their utterance (Beattie, 
1978; Goldman-Eisler, 1967; Kendon, 1967), or during discussion of more difficult topics (Ellyson, Dovido, \& Corson, 1981; Exline \& Winters, 1965). Should they fail to avert their gaze at such times, they produce more false starts (Beattie, 1978, 1980, 1981). Because each listener in a dialogue is a potential speaker, planning utterances while listening, each has ample reason not to keep the other's face in view.

Finally, interlocutors may simply have a more pressing need for other visual information. When visible objects are relevant to the conversation, only $3 \%$ to $7 \%$ of the conversation time is accompanied by gaze of one participant at another (Argyle, Alkema, \& Gilmour, 1972; Argyle \& Graham, 1977; Krantz, George, \& Hursh, 1983).

These findings suggest that, in quiet conditions at least, there are natural limits to the uptake of visual aids to speech perception or message understanding. However useful it might be to track listeners' facial expressions, to watch articulatory movements, or to see whether one's own face is being monitored, visual cues may nonetheless be used only intermittently in face-to-face dialogue.

It is this restriction that allows us to look for coarsegrained and fine-grained influences on speakers' articulation. In the following experiments, we examine word tokens excerpted from a corpus of task-related dialogues conducted in a recording studio. Although partners were always facing each other, only the subjects in half these dialogues had a clear view of their partners' faces; subjects in the other half of the design had obscured lines of sight. If visual information can be recruited to the task of deciphering speech, we should find at least a coarsegrained effect of visual information: Speakers with clear lines of sight should articulate less carefully than their screened counterparts.

Note that this first prediction could be fulfilled without speakers' taking careful account of the moment-tomoment state of the listeners' knowledge. Fine adjustment of articulatory quality to the uptake of facial cues demands that speakers and listeners look at one another while they speak and that intelligibility changes are associated with the appropriate direction of gaze. Because it is unlikely that speaker and listener will gaze at each other constantly, there will be occasions within those dialogues performed with clear sight lines when speakers can be seen to observe listeners looking at them, and others when both participants are looking at the task materials instead. If the control of articulation depends on a close account of the listener's knowledge, then speakers will be less careful only while they can see that listeners are actually looking at them.

\section{EXPERIMENT 1}

Experiment 1 tested the hypothesis that speakers react to the presence of a visual channel by speaking more casually. The experiment compares intelligibility for tokens of the same word produced under nearly identical circumstances by speakers whose partners could or could not see them. If visual and auditory cues to word identity are integrated, tokens should be less intelligible when the listener can check for both kinds of cues.

\section{Method}

\section{Materials}

Because the effects of redundancy on intelligibility are greatest in extended speech used for real communication (Fowler, 1988), all materials were taken from a corpus of unscripted dialogues recorded while pairs of speakers attempted a collaborative problemsolving task. The HCRC Map Task Corpus (Anderson et al., 1991) consists of 128 such dialogues.

Task. The corpus uses the map task devised by Brown, Anderson, Shillcock, and Yule (1984). In this task 1 subject, the instruction giver, has a schematic map with a route printed on it that can be described relative to a set of labeled landmarks. The other subject, the instruction follower, has a similar but not identical map without a route. Neither subject can see the other's map. The two collaborate at whatever length they require to reproduce the giver's route on the follower's map, with no restrictions on what either can say or on who controls the agenda of the dialogue at any point. Subjects were warned that the maps might not be the same in every respect. In fact, matching landmarks alternated with mismatched landmarks in various ways: Some were missing on one or the other map, some occurred twice on one map and once on the other, and some were labeled differently on the two maps.

Though quite demanding, the map task is engaging for both givers and followers, producing unself-conscious dialogues that ranged from 3 to $9 \mathrm{~min}$ in length. Extract 1 shows the start of a typical map task dialogue.

Extract 11

A: Ready Dave?

B: Yeah, fire away.

A: Right. Starting, above the site of the forest fire.

B: Certainly am.

A: Right. Go east ... west sorry. I don't know what I'm talking about.

Go west slightly, just past it and down and then you go south.

B: And go... hang a bit left right past it? Right.

A: So you... so you're going east west then south, down.

B: Yup.

A: Have you got a pienic site?

Speakers. Sixty-four undergraduates at the University of Glasgow (32 male and 32 female) with a mean age of 20 took part. Sixty-one of the subjects were Scottish, 56 of them born and brought up within a 30 -mile radius of Glasgow. Two were English and 1 was American. All were native speakers of English.

Procedure and Design. Map tasks were performed in a recording studio, with subjects sitting 3-4 feet apart and facing each other across a sort of double drafting table where each subject's side supported his/her map and sloped toward the subject steeply enough to block any view of the other map.

In half the dialogues, each speaker could see the partner's head and shoulders (face visible). Map and chairs were arranged so that changing direction of gaze from the map to the partner's mouth required a vertical eye movement of $12^{\circ}$ at the top of the map and $60^{\circ}$ at the bottom. In the other half of the dialogues, a screen of flimsy cardboard was placed at the apex of the sloping table surfaces about 2 feet from the subjects and adjusted to a height that just obscured the subjects' faces (face screened). Pairs of subjects who were well known to each other were randomly assigned to a face condition with another pair whom they first met at the start of the recording session. Each quadruple of subjects produced a sequence of eight dialogues within which each subject took part in four dialogues, counterbalancing role and partner familiarity. 
The first four dialogues used new pairs of maps each time. The second four allowed instruction givers to use a map they had already described, but with a different follower. The screened and visible conditions had identical designs. Eight quadruples of subjects and eight sets of maps were run in each condition. After performing the map tasks, each subject was asked to read carefully a list containing all the words used as landmark labels for their set of maps. This meant that for each target word in running speech, there was a corresponding citation form from the same speaker available for comparison. More details of the design of the corpus are given in Anderson et al. (1991).

All materials were digitally recorded on DAT (Sony DTC1000ES) using one Shure SM10A close-talking microphone and one DAT channel per subject. Split-screen video recordings were also made for half the dialogues, with a video camera (JVC TK 8803) placed behind each subject to capture an almost full-face image of the other. Full orthographic transcriptions were made of all the dialogues by experienced transcribers and subsequently checked 5 to 10 times against the original DAT recordings.

\section{Stimuli and Design}

Stimuli were selected from map task dialogues with controls for conditions that are known to affect intelligibility as measured on excerpted word tokens. All spontaneous stimuli were single open class words appearing in names of landmarks printed on maps. A second citation token of the same name always came from the reading of landmark lists by the same speaker at the end of the series of dialogues. All the named landmarks were visible to the speaker when the spontaneous token of the name was uttered in the course of a dialogue. To test for the effects of the listener's access to the speaker's face, each word form had to be used spontaneously both by a speaker who was visible to his/her listener and by another who was not. For 70 of the 72 word pairs, exactly the same map variant was being used by the visible and the screened speaker. For the remaining two items, the words named identical landmarks on slightly different maps. Because intelligibility is known to decrease between first and second mentions, each spontaneous token was both the first token of the word form used to refer to a landmark in its dialogue and the first mention of that landmark in any terms. So that no previous information was available to the speaker about the utility of the term or the landmark in the task, all dialogue tokens were from the first dialogue in which the speaker played his/ her current role for a particular map. Finally, to control for phonetic context between spontaneous and read tokens, all stimuli had to be uttered within a full literal rendition of the landmark name. The complete set of 72 items used in identical conditions by facevisible and face-hidden speakers comprised all the tokens of landmark names in the corpus that met these conditions and that were not disqualified by dysfluency or overlap with the other speaker

For 48 of these items, another condition was also met. For purposes of another experiment, these words also had to be reintroduced in the same speaker's second attempt at giving instructions on the same map. Accordingly, we can assume that these words proved useful in the communication of the route. Thus, for example, from Extract 1 we could select the individual nouns within the two landmark descriptions site of the forest fire and picnic site if Speaker A later used the same full phrase to introduce the same landmark when giving the same instructions to a new listener and if some other informant in a complementary face condition made a comparable introduction of the term. The remaining 24 words referred to landmarks that either were not reintroduced in a second giving of the same map or were not reintroduced by usable tokens. Under these criteria, the same items from Extract 1 would have been acceptable regardless of their subsequent use.

Each of the 72 word forms yielded four word tokens - one spontaneous and one citation token each from one speaker in the facevisible condition and another in the face-screened condition. Each utterance containing 1 of the 144 spontaneous word tokens and each full landmark name containing the corresponding citation form was sampled from the appropriate channel of the original DAT recordings at a rate of $16 \mathrm{kHz}$ using the Entropic Signal Processing System through the XWAVES speech analysis program. The average amplitude of the critical materials was adjusted to remain as constant as possible across stimuli. The start and end points of each word were located using a combination of auditory information and visual inspection of the WAVES time-amplitude waveforms and broadband spectrograms. For the purposes of other experiments, spontaneous tokens produced when the same items were reintroduced in later dialogues were digitized in the same way, as were other word tokens from the same corpus.

To avoid ceiling effects in intelligibility, excerpted word tokens were multiplied by noise. A $16-\mathrm{kHz} 5-\mathrm{sec}$ file of random noise (in which all sample values were in the range $0.5-1.5$ ) was created. This was multiplied by each original speech file, sample by sample, to create an output file the amplitude of which was related to that of the original speech and the data points of which had the same signs as the sampled data values they replaced. The relationship between signal and noise is best described by the range of noise values, rather than by a signal-to-noise ratio.

The noise-overlaid speech data files were copied onto DAT with an interstimulus interval (ISI) of $8 \mathrm{sec}$. Word tokens were allocated to different presentation tapes according to a Latin square design. Because the tapes included further tokens of the same words for a different experiment, there were six tapes rather than four. The order of presentation of items was randomized, with the same random order of words adopted for each tape.

\section{Subjects and Procedure}

Subjects were undergraduates at the University of Glasgow and members of the subject population sampled in the corpus that provided the stimuli, though none had been corpus informants. All subjects were native speakers of some variety of British English, had no reported hearing loss, and were paid for their participation. Subjects were run in groups of varying sizes but each heard the stimuli over headphones while sitting in a separate booth and typing responses directly onto disk.

Subjects were instructed that all the stimuli were real English words but that some might be difficult to identify. They were asked to listen carefully and to guess if they were not sure which word was said.

Subjects were allocated randomly to one of six presentation tapes, with 9 subjects hearing each tape. The same four practice items occurred on all tapes.

\section{Results and Discussion}

\section{Face Visibility}

Intelligibility. One stimulus item was lost because of experimenter error, leaving 71 word forms with four tokens each. Subjects' attempts to recognize these were scored as correct if they were accurate spellings of the stimulus words or of their homophones (beaches for beeches) or if they were misspelled but unmistakable (machetti for machete) ${ }^{2}$

Scores for correct recognition were then submitted to analyses of variance (ANOVAs) $\times$ subjects and materials. In one pair of ANOVAs, the dependent variable was raw intelligibility, the proportion of correct identifications of the excerpted word token. Token (spontaneous vs. citation) was crossed with dialogue condition (face visible vs. face screened) and both were repeated measures. For each raw intelligibility analysis, a matching analysis of 
intelligibility loss was also performed. Here the dependent variable was the difference in rate of correct identifications between careful citation tokens and spontaneous introductory mentions. These analyses have the advantage of removing differences in baseline citation form intelligibility from consideration and focusing the tests directly on changes in clarity. We will present the results primarily through the analyses of intelligibility loss, referring to analyses of raw data only when baselines are at issue.

Dialogue condition had an effect on the intelligibility of spontaneous word tokens. As Figure 1 shows, intelligibility loss from citation to spontaneous form was significantly greater for face-visible dialogues $(23 \%)$ than for face-screened dialogues $(10 \%)$ [ for the main effect of dialogue condition, $F_{1}(1,53)=24.21, p<.0001 ; F_{2}(1,70)=$ $\left.5.95, p<.02 ; \operatorname{Min} F^{\prime}(1,101)=4.78, p<.05\right] .{ }^{3}$ ANOVAs on raw intelligibility showed that the effect of dialogue type was due to the low intelligibility of the spontaneous word tokens from face-visible dialogues. In fact, facevisible spontaneous tokens ( $59 \%$ correct) were less intelligible than either their own citation forms $(82 \%)$ or the introductory tokens of the same words in the face-screened condition $(74 \%$; Scheffé tests by both subjects and materials at $p<.01$ ). Unlike the spontaneous tokens, the citation forms from face-visible $(82 \%)$ and face-screened $(84 \%)$ conditions did not differ significantly.

Duration. ANOVAs $\times$ materials were also run on the durations of excerpted word tokens and had the same design as the materials intelligibility analyses. Although word durations did not support a significant effect $\left[F_{2}(1,70)\right.$ $=2.57, p=.11]$, the numerical differences were in the same direction as they were for intelligibility, with citation forms of extremely similar duration (face visible,

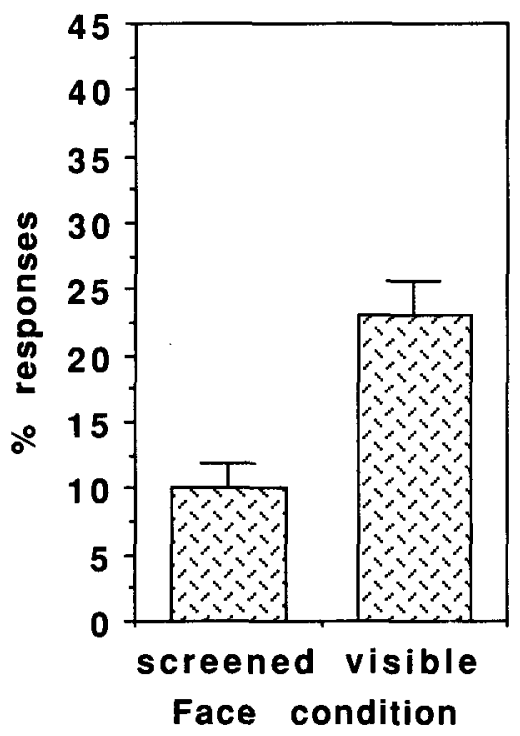

Figure 1. Effects of face visibility on intelligibility loss in Experiment 1 (recognition rate for citation forms - recognition rate for spontaneous forms taken from running speech) expressed as percent of responses.
$530 \mathrm{msec}$; face screened, $531 \mathrm{msec}$ ) and spontaneous face-screened introductions $(423 \mathrm{msec})$ longer than their spontaneous face-visible $(397 \mathrm{msec})$ counterparts.

Speakers in this experiment appeared to be treating visual cues as contributors to the recognition of running speech. Words produced with the interlocutor's face accessible behaved like words produced with useful discourse and extralinguistic context: They were said less clearly. The fact that routes of equal accuracy (Boyle et al., 1994) are produced in face-screened and face-visible dialogues tells us that on a gross level, the different distributions of information produce equally successful communication.

Gross adjustments, however, are only the preliminary conditions for concluding that speakers tune their delivery to close observation of listeners' perceptual needs. To determine whether the results are linked to the uptake rather than the availability of visual cues, we must know more about where speaker and listener were looking in the face-visible dialogues and whether the direction of gaze was associated with changes in intelligibility.

\section{The Incidence of Gaze in Face-to-Face Dialogue}

A sample of the HCRC Map Task Corpus. The literature on gaze in conversation, particularly in visually rich environments, suggests that listeners may not use the visual cues available in face-to-face communication frequently enough to optimize word recognition. To determine whether the results of Experiment 1 could be attributed to the use of such cues, we first asked whether the speakers and listeners of the HCRC Map Task Corpus actually looked at each other.

To answer this question, the 32 face-visible dialogues (out of 64) that had been videotaped were comprehensively coded for gaze by two coders who were unaware of the nature of subsequent analyses to be performed on their data. Each coder viewed the videotapes with a full transcription of the dialogue and marked each word or pause during which either subject looked at the other. To test interjudge reliability, a randomly chosen sample dialogue was coded independently. The coders agreed in their with/ without gaze classification on $97 \%$ of the 941 words of this dialogue. To test intrajudge reliability, coders also recoded a dialogue they had analyzed some weeks earlier. One coder agreed with her own previous judgments on $94 \%$ of words, the other on $92 \%$.

For each of the 32 dialogues, separate counts were made of words accompanied by speaker, listener, or mutual gaze. The first line of entries in Table 1 shows the summary figures. Across all the dialogues, an average $30.2 \%$ (range, $10.8 \%-47.4 \%$ ) of words were accompanied by speaker or listener gaze. Some $12.9 \%$ of words (range, $0.6 \%-37.6 \%$ ) were said while their speaker was looking at their listener and $17.3 \%$ (range, $1.3 \%-46.1 \%$ ) while the listener was looking at the speaker.

Although the ranges on these counts indicate that some subjects had ample opportunity to discover the visual cues to speech sounds, speakers rarely made use of the oppor- 
Table 1

Percent of Word Tokens in Task-Oriented Dialogue Accompanied by Speaker, Listener, and Mutual Gaze

\begin{tabular}{|c|c|c|c|c|c|}
\hline \multirow[b]{2}{*}{ Source } & \multicolumn{5}{|c|}{ Gaze Type } \\
\hline & $N$ & Speaker & Listener & Mutual & Total \\
\hline 32 HCRC Map Task & 36,990 & 12.9 & 17.3 & 2.7 & 30.2 \\
\hline Face-visible dialogues & & $(0.6-37.6)$ & $(1.3-46.1)$ & $(0-9.4)$ & $(10.8-47.4)$ \\
\hline $\begin{array}{c}\text { Subsample } 1 \text { : Coded face-visible } \\
\text { materials from Experiment } 1\end{array}$ & 43 & 18.6 & 11.6 & 2.3 & 30.2 \\
\hline $\begin{array}{l}\text { Subsample 2: Coded face-visible } \\
\text { materials from earlier experiments }\end{array}$ & 295 & 12.5 & 10.5 & 2.0 & 23.0 \\
\hline
\end{tabular}

tunity to observe listeners picking up these cues: Mutual gaze was consistently rare in all 32 dialogues, with a mean of $2.7 \%$ of words and a range from $0 \%$ to $9.4 \%$. The observations on mutual gaze are broadly in line with the literature on gaze in conversations where a concurrent task requires visual information $(3 \%-7 \%)$, although the observations on individual gaze are not unusual in view of the social psychological literature as a whole.

Extract 2 shows a fairly typical pattern and incidence of gazing in the map task dialogues. The speaker was looking at the listener while saying the words in italics. The listener was looking at the speaker during the words in bold. Speaker and listener looked at each other during the words that are both bold and italicized.

Extract 2

A: < Could you move down straight down from there... have you got a haystack/

B: Yeah

A: On your map? Right just move straight down from there then /

B: Past the blacksmith?>

A: Past the haystack to eh have you got a carpenter's house?

B: Yeah right, I know where you are

A: <parallel with the bottom of the ... the

B: Right

A: Carpenter's house $>$ OK right could you move across...

A subsample of Experiment 1 materials. The figures for the gaze-coded dialogues as a whole give a good account of the conditions under which the materials in Experiment 1 were said. The 32 coded dialogues contained 43 of the 71 face-visible spontaneous word tokens used as stimuli in Experiment 1. The second line of figures in Table 1 shows the results for this subsample of map task materials. Here a total of $30.2 \%$ of words were said with speaker or listener gaze, $18.6 \%$ while the speaker was looking at the listener, $11.6 \%$ while the listener was looking at the speaker, and $2.3 \%$ while each looked at the other.

Again, the low incidence of gaze in these materials, particularly of mutual gaze, makes it difficult to attribute the results of Experiment 1 to speakers' close appreciation of the listeners' visual resources. To test the utility of gaze itself, we must compare the intelligibility of words spoken in face-visible dialogues in which speaker and listener gaze did or did not occur.

\section{MATERIAL FROM EARLIER EXPERIMENTS}

\section{Method}

Materials

Existing materials the intelligibility of which was known made it possible to pursue the relationship between gaze and intelligibility. Words had been drawn from the HCRC Map Task Corpus for a series of intelligibility experiments conducted in parallel to the studies described in this paper but exploring different variables (Bard, Sotillo, Anderson, Doherty-Sneddon, \& Newlands, 1995). In all cases, the procedure for preparing speech materials, the instructions to subjects, and the subject population sampled were the same as those described for Experiment 1 . The number of subjects attempting to identify each word in any one experiment always ranged from 9 to 18 .

A post hoc search collected all the stimuli of known intelligibility that had been extracted from the gaze-coded dialogues. This sampling produced 295 word tokens spoken in face-visible dialogues. The third line of data in Table 1 shows the incidence and pattern of gaze in this adventitious sample. Of the 295 words, $23 \%$ were accompanied by gaze, $12.5 \%$ with speaker gaze, $10.5 \%$ with listener gaze, and only $2 \%$ were said while the speaker and listener looked at each other. Comparison with the rest of the table shows that these materials and the others are similar in distribution.

\section{Design}

To explore the effect of gaze on clarity, we used any word from an earlier experiment that was accompanied by speaker or listener gaze and that could be paired with another word token uttered by the same speaker in a face-visible dialogue at a point when no gaze occurred. The paired words were matched for original position in the relevant noun phrase, for referential role (introduction of landmark or repeated mention), and, if the word was a repeated mention, for the identity of the speaker who first introduced it. These necessary controls precluded examining the effects of gaze within words and within speakers at the same time: A speaker has only one opportunity to mention a landmark for the first time, during which gaze is either directed to the other speaker or not. Of the 295 word tokens used in earlier experiments, 52 were accompanied by gaze and could be paired with suitable nongaze words within these same materials. Of these, 25 were introductory mentions and most closely analogous to the face-visible items of Experiment 1 . For all 52 suitable items, we examined the difference between the observed intelligibility scores for spontaneous and citation forms. Again, comparisons of duration provided an auxiliary measure.

Mutual gaze, which would best ensure that the speaker could notice the listener's gaze, was too rare to use as the working definition of gaze. In the physical arrangement used for conducting map task dialogues, either subject's change of gaze could have created a discernible movement if only in the periphery of his/her part- 
ner's visual field. ${ }^{4}$ To test for the use of the subtle signals available in this way, we contrasted words with no gaze and words with any gaze, whether by speaker or listener.

\section{Results}

\section{Intelligibility}

Intelligibility was defined here as the proportion of listeners able to identify the word token correctly over all the experiments in which it had been used. The major dependent variable was once more intelligibility loss-reduction in intelligibility from the corresponding citation form.

Analyses of subjects were inappropriate herre. An ANOVA on materials for gaze (present, absent) $\times$ role of gazer (speaker, listener) $\times$ spontaneous token (first mention, second mention) used word pairs as the unit of analysis. Gaze was a repeated measure whereas role and token were between items. The effect of gaze on intelligibility loss was not significant. Intelligibility loss was $22.4 \%$ with gaze and $25.9 \%$ without gaze $\left(F_{2}<1\right)$. For the 25 introductory mentions alone (the items most similar to those showing an effect of face visibility in Experiment 1), the losses were $10.2 \%$ with and $26.2 \%$ without gaze [in a gaze $\times$ gazer ANOVA for first mentions: $F_{2}(1,23)=$ $3.82, p=.06]$. That is, the results approached significance but in the direction opposite to our prediction. The interaction of gaze with role of gazer was not significant in either case $\left(F_{2}<1\right.$ in both analyses).

\section{Duration}

A second ANOVA, identical in design to the intelligibility analysis just described, was used to examine the effects of gaze, gazer role, and token on changes in duration from citation form to running speech token. Once more, there was no significant effect of gaze on the degradation of running speech forms: With-gaze words were reduced by $151 \mathrm{msec}$, without-gaze by $126 \mathrm{msec}\left[F_{2}(1,48)=1.33\right.$, $p>.20]$. Citation forms were comparable in length, averaging $506 \mathrm{msec}$ with gaze and $501 \mathrm{msec}$ without. First mentions considered alone also showed no effect of gaze, being reduced by $158 \mathrm{msec}$ with gaze and $124 \mathrm{msec}$ without [from citation forms of 534 and $522 \mathrm{msec}$, respectively; $\left.F_{2}(1,23)=1.76, p=.20\right]$.

\section{Discussion}

The outcome of this post hoc study does not allow us to attribute the results of Experiment 1 to fine-grained use of visual cues. There were no significant effects of gaze on intelligibility or duration. The nearest the results came to significance was an intelligibility effect $(p<$ $.10)$ in the wrong direction: greater loss of intelligibility with gaze for first mentions.

It is possible, however, that this study was subject to an artifact. Experiment 1 matched words across face conditions, comparing paired tokens of the same landmark names from different speakers who could or could not see their listeners. It produced a significant effect of face visibility within word form. The post hoc study, on the other hand, did not match words across gaze conditions. Instead it used similar but not identical words produced by the same speaker. It failed to find the predicted effect of gaze. If effects are reliable only when materials match across the variable of interest, we need to look for a gaze effect using tokens of the same words produced with and without gaze. For this design, we turn to Experiment 2.

\section{EXPERIMENT 2}

\section{Method}

\section{Materials}

From the gaze-coded face-visible dialogues, 60 word forms were selected. Each had an introductory full literal mention from 2 speakers, one with and the other without gaze. With-gaze tokens were of two types: 30 of the 60 were said while the speaker was watching the listener; the rest were said while the listener was watching the speaker (either with $[n=9]$ or without $[n=21]$ simultaneous speaker gaze). These tokens and their corresponding citation forms, 240 items in all, were multiplied by noise, as in Experiment 1 , and distributed across four tapes in a Latin square design.

\section{Subjects and Design}

Seventy-two undergraduates at the University of Glasgow acted as subjects in this experiment. Eighteen subjects heard each stimulus tape, so that each listener heard each of the 60 words in one and only one token (spontaneous, citation) $\times$ gaze (with, without) condition and each heard equal numbers of items from each cell of the design. The instructions and listening conditions were the same as those described in Experiment 1.

\section{Results}

\section{Intelligibility}

ANOVAs were conducted for $\times$ subjects and $\times$ materials. For subjects, gaze and role of gazer were repeated measures. Materials were nested within the level of gazer role (speaker, listener) appropriate for the with-gaze spontaneous token of each pair, whereas gaze remained a repeated measure. In all cases, the results are quoted from ANOVAs on the intelligibility loss measure, and analyses of raw intelligibility (gaze $\times$ gazer $\times$ form) were used to check for artifacts of variation in control forms.

Figure 2 a shows that, contrary to prediction, intelligibility loss was greater for tokens introduced without gaze $(23 \%)$ than for tokens produced with gaze $(16 \%)$ $\left[F_{1}(1,68)=5.09, p<.03 ; F_{2}(1,57)=1.28, p>.10\right]$. Figure $2 b$ shows that the result held only for items uttered with listeners' gaze [Scheffé at $p<.01$ within both gaze $\times$ gazer role interactions; $F_{1}(1,68)=42.67, p<.0001$; $\left.F_{2}(1,58)=6.25, p<.02 ; \operatorname{Min} F^{\prime}(1,75)=5.45, p<.025\right]$.

\section{Duration}

Analyses of duration loss also used difference between citation and running speech forms as a dependent variable and followed the materials design for intelligibility loss. As Figure 2c shows, the effect of gaze was once more contrary to prediction: The $128-\mathrm{msec}(26 \%)$ reduction for without-gaze items was significantly larger than the 93msec $(19 \%)$ difference for with-gaze items $\left[F_{2}(1,58)=\right.$ 

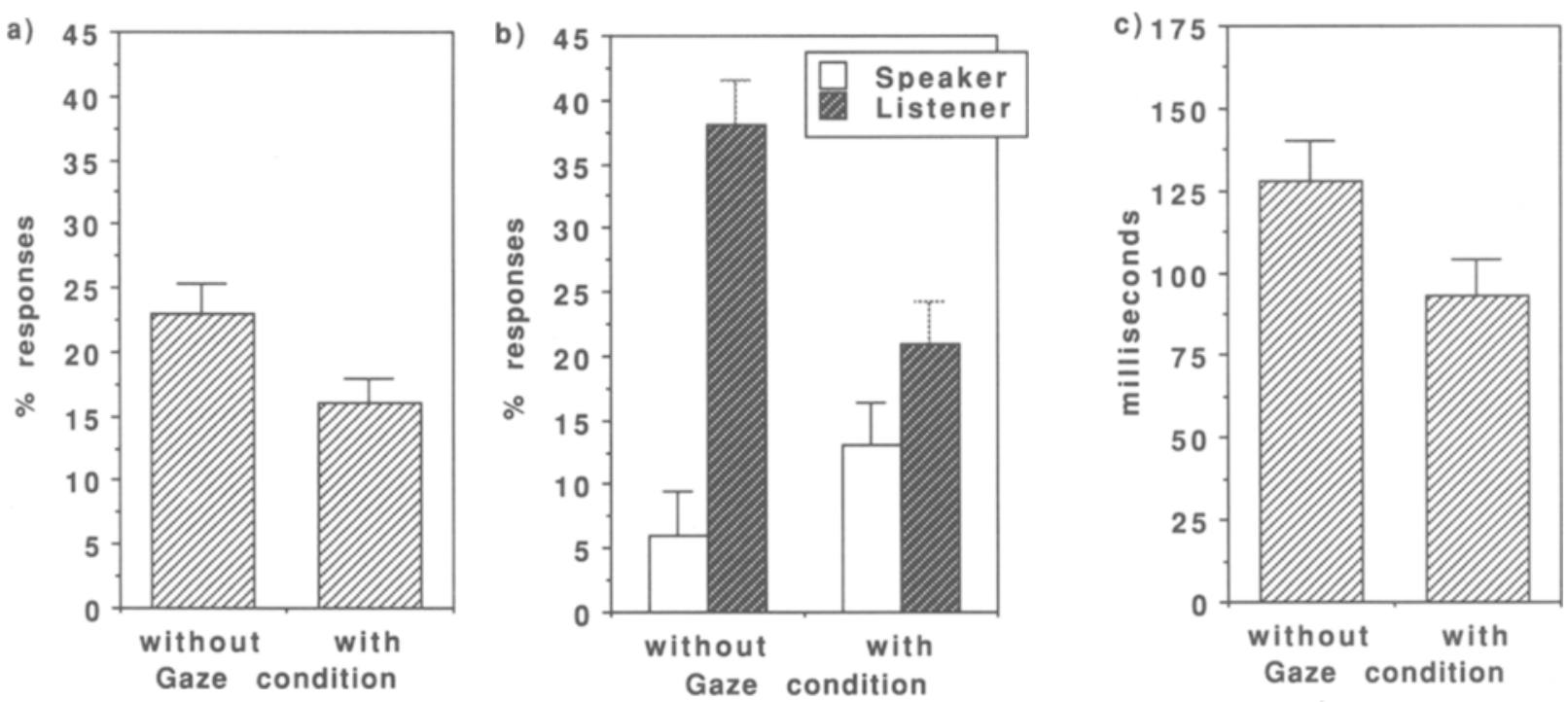

Figure 2. For Experiment 2, the (a) effects of subject gaze on intelligibility loss (in percent of responses); (b) effects of gaze and of gazer role on intelligibility loss; and (c) effects of gaze on duration loss (in milliseconds).

$5.04, p<.03$ ]. Citation forms were comparable in length (498 msec without gaze, $489 \mathrm{msec}$ with). The interaction between gaze and gazer role was not significant $\left(F_{2}<1\right)$.

\section{GENERAL DISCUSSION}

In the work reported here, our purpose was to discover how closely speakers model the resources that listeners can bring to bear on the recognition of running speech. The results confirm earlier evidence (Bard \& Anderson, 1983, 1994; Bard et al., 1989) suggesting that speakers maintain very loose models of the information available to listeners. Although speakers articulate less carefully in ambient visual conditions that might help the listener, we find no evidence to suggest that they track the listener's use of visual information from moment to moment or that they articulate less carefully only when they observe the listener exploiting such information.

Experiment 1 showed a robust but gross adjustment: Speakers introduce new objects into a dialogue less intelligibly if speaker and listener can see each other's faces. If tracking use of the visual medium was necessary to the overall control of intelligibility, subjects should have been looking at each other much of the time. Yet the word-byword account of gaze direction revealed that less than one word in three was uttered while one subject was actually looking at the other's face. A selection of words used in earlier experiments did not appear to be articulated with less care when either speaker did look at the other. With a new selection of matching word forms, Experiment 2 also gave no indication of decreasing articulatory care with local increases in use of the visual channel. Instead, speakers articulated with significantly greater care when listeners looked at them.
The effect of actual access to the interlocutor's face (Figure 2a) was smaller than the effect of potential access (Figure 1). Nonetheless, intelligibility loss for the withoutgaze materials, which were the more typical in facevisible dialogues, was close to the overall figure $(23 \%)$ for face-visible materials in Experiment 1 (Figure 1). Whatever the results of Experiment 2 mean, there is no reason to think that they fail to represent the population of words in face-to-face dialogue.

If a model of spoken communication in which all available information is monitored and adjusted to will not accommodate these results, what will? Why do map task subjects look at one another so rarely in comparison with subjects in lip-shape experiments using carefully articulated speech? What conditions loss of clarity for face-visible dialogues if not a local visual assessment of the listener's input? Why do speakers articulate more clearly when their listeners are using the visual channel? The answers to these questions appear to lie in the role of visual information in face-to-face communication.

First, why do speakers fail to monitor each other's faces for cues to articulation? For every laboratory study showing that normal adults can make use of lip shape in speech recognition, there seems to be one showing that they look at each other too rarely to use lip shape on a regular basis. The subjects in the map task corpus were certainly not looking at each other whenever their physical circumstances permitted. Even deaf lip readers, for whom visual cues are absolutely critical, experience difficulty maintaining a constant view of the speaker's face, especially when engaged in a concurrent visual task (Mogford, 1987). For hearing individuals, the motivations for not gazing at an interlocutor may simply outweigh the motivations for attending to lip shape. Like the present results, 
the conflict between the social and perceptual literatures reminds us that the potential resources for the perception of speech may not all be exploited in particular communicative contexts. We already know that spontaneous running speech differs from scripted linguistic material in the way speakers control pronunciation and in the cues used by listeners in process speech (Fowler, 1988; McAllister, Potts, Mason, \& Marchant, 1994; Mehta \& Cutler, 1988). To predict whether people will actually use particular resources, we will need more extensive studies of the factors bearing most strongly on the recognition of speech in dialogue.

At this point, we ought to consider whether the demands of the map task itself might make our corpus the wrong one to search for effects of visual information. Modeling a listener's understanding is, we have suggested, a difficult task in optimal circumstances. When absorbed in collaborative problem solving, our speakers might simply have been too busy looking at their maps to use their eyes in speech perception or to manage a synchronized tradeoff between the listener's uptake of visual cues and the clarity of their own articulation.

Several facts argue against this view. First, the levels of gaze found in the map task corpus were not unusually low. They were well within the range observed in social psychological studies, especially among those studies with no distracting visual tasks. Only mutual gaze was extremely and consistently rare. Second, if keeping eyes on the maps had been of such paramount importance that no practical use could be made of other visual information, there should have been no discernible differences between face-visible and face-screened dialogues. Yet such differences were found. One of them was the intelligibility effect of Experiment 1. Others, reported by Boyle et al. (1994) and by Doherty-Sneddon et al. (in press), were summarized earlier. Map task subjects who had clear sight lines used occasional views of one another's faces, usually to check the health of the communication or to supplement verbal descriptions that were proving hard to interpret. Third, since gaze replaced certain kinds of utterances, subjects who were too preoccupied with their maps to receive gaze-borne information should have suffered from relatively impoverished feedback. They ought to have produced either more faulty routes or more iterations to get them right. Instead they were as accurate as face-screened pairs using the same maps, and faster overall. All the indications are that the visual channel was used effectively in the map task corpus.

Now we can return to the question of intelligibility. If speakers articulated less carefully when introducing new landmarks in face-visible than in face-screened dialogues, and if the effect was not promoted by the transmission of lip shape, of facial expression, or even of the information that these were being monitored, then what did condition it?

Our best guess is that subjects in face-visible dialogues adjusted their articulatory precision to a global impression that they were communicating well and easily. The relatively rare episodes when subjects examined their partners' faces were not responsible. The more common condition, in which neither subject used this channel (Figure 2a), produced characteristic intelligibility of these dialogues as a whole (Figure 1). In these passages, speakers may have been basing their impressions on their ability to continue the dialogue without interruptions or back channels. They may have succumbed to the illusion that they were actually being viewed when it was merely the case that they could be viewed. They may well have assumed that without visual signs of difficulty, their own model of the discourse and their listener's model were fully aligned.

By the same token, subjects in face-screened dialogues might have harbored an impression of strain, since they simultaneously spoke and monitored for the listener's contributions or restarted after interruptions. They may also have articulated more clearly, as we would originally have predicted, because they realized that the auditory channel is critical when the visual channel is unavailable. In any case, we would suggest that speakers in the two conditions were adjusting care in articulation to complement a global impression of the apparent margin of safety for the transmission of their messages, rather than a frequently refreshed account of the listener's state.

Given the evidence for coarse tuning, what are we to make of the surprising effects of gaze on word duration? On those relatively rare occasions when listeners looked at speakers, speakers supplied more auditory detail, acting as if listeners were short of information, not as if lip shape and interactive cues provided it in abundance. Given what we know about interactive facial information in dialogue, their behavior is not unreasonable. If gaze is most frequent at points at which communication is difficult, it can function as a "poor health" bulletin for the developing dialogue. A speaker estimating that the shared discourse background is in doubt might well be inclined to speak more deliberately.

These results suggest a complex system of influences on care in articulation. Speakers may respond to global conditions without devoting much attention to maintaining a delicate Gricean link between articulatory clarity and the moment-to-moment flow of visual cues to word identity. Instead, their local adjustments are made only in response to overt visual signals on the part of their interlocutors. Fully developed models of speaker and hearer will have to explain what visual information, potential or real, affects the delivery and the perception of speech. With this addition, our models of the control of articulation will enter the domain of current theories of dialogue and discourse, showing how speakers represent ambient information and attribute particular knowledge to their listeners. Models of speech perception must also reflect the limitations on listeners' monitoring capacities. Even incomplete, such models will serve a practical need as an adjunct to a developing technology.

At present, multimedia communication systems are in advanced stages of development, but some appreciation of the perceptual facts must underpin design features that will make them usable. Augmenting audio communication 
with videophones and videowindows may be counterproductive unless the technology permits the normal interaction between visual information and the control of speech production. In fact, users often find dual-modality systems more difficult than familiar audio links (O'Malley \& Langton, 1994). Aside from the McGurk effects that can arise from poorly synchronized video and audio, we might now predict additional difficulties if speakers articulate less clearly in the videolinked dialogues. To compensate, the technology may need to provide better, not worse, sound reproduction with video links. Moreover, video images may have to change swiftly enough to allow concurrent speaking and monitoring for comprehension. Late or irregular updates may fail to give speakers the impression that they are looking at one another and so may also fail to elicit the more careful articulation that we find with interspeaker gaze. Although we do not yet know how people will react to particular exemplars, successful multimedia communication will most probably be achieved by systems that help speakers use visual information to model the progress of their conversations.

\section{REFERENCES}

ABELE, A. (1986). Functions of gaze in social interaction. Journal of Nonverbal Behaviour, 10, 83-101.

Anderson, A. H., Bader, M., Bard, E. G., Boyle, E., Doherty, G., Garrod, S., Isard, S., KowtKo, J., McAllister. J., Miller, J., Sotillo, C., Thompson, H. S., \& WeinerT, R. (1991). The HCRC Map Task Corpus. Language \& Speech, 34, 351-366.

ARGYL, M. (1990). Bodily communication. London: Routledge.

Argyle, M., Alkema, F., \& Gilmour, R. (1972). The communication of friendly and hostile attitudes by verbal and non-verbal signals. European Journal of Social Psychology, 1, 385-402.

ARgYle, M., \& DeAN, J. (1965). Eye-contact, distance and affiliation. Sociometry, 28, 289-304.

Argyle, M., \& Graham, J. A. (1977). The Central Europe experiment: Looking at persons and looking at things. Journal of Environmental Psychology \& Nonverbal Behaviour, 1, 6-16.

ARGYLE, M., \& INGHAM, R. (1972). Gaze, mutual gaze and proximity. Semiotica, 6, 32-49.

ARIEL, M. (1990). Accessing noun-phrase antecedents. London: Routledge/Croom Helm.

BARD, E. G., \& ANDERSON, A. H. (1983). The unintelligibility of speech to children. Journal of Child Language, 10, 265-292.

BARD, E. G., \& ANDERSON, A. H. (1994). The unintelligibility of speech to children: Effects of referent availability. Journal of Child Language, 21, 623-648.

Bard, E. G., Lowe, A., \& Altmann, G. (1989). The effects of repetition on words in recorded dictations. Proceedings of EUROSPEEC $H$ '89, 2, 573-576.

Bard, E. G., Sotillo, C., Anderson, A. H., Doherty-Sneddon, G., \& NEWLANDS, A. (1995). The control of intelligibility in running speech. Proceedings of the XIIIth International Congress of Phonetic Sciences, 4, 188-191.

BeATtIE, G. W. (1978). Sequential patterns of speech and gaze in dialogue. Semiotica, 23, 29-52.

BEATTIE, G. W. (1980). The role of language production processes in the organization of behaviour in face-to-face interaction. In B. Butterworth (Ed.), Language production: Vol. 1. Speech and talk (pp. 69-107). London: Academic Press.

Beatrie, G. W. (1981). A further investigation of the cognitive interference hypothesis of gaze patterns in conversation. British Journal of Social Psychology, 20, 243-248.

BEATtIE, G. (1983). Talk: An analysis of speech and non-verbal behaviour in conversation. Milton Keynes, U.K.: Open University Press.
BOLINGER, D. (1963). Length, vowel, juncture. Linguistics, 1, 5-29. BOLINGER, D. (1981). Two kinds of vowels, two kinds of rhythm. Bloomington: Indiana University Linguistics Club.

Boyle, E. A., Anderson, A. H., \& Newlands, A. (1994). The effects of visibility on dialogue performance in a cooperative problem solving task. Language \& Speech, 37, 1-20

Brown, G., Anderson, A. H., Shillcock, R., \& Yule, G. (1984). Teaching talk. Cambridge: Cambridge University Press.

ChafE, W. (1974). Language and consciousness. Language, 50, 111 133

Doherty-Sneddon, G., Anderson, A. H., O'Malley, C., LangTON, S., GARROD, S., \& BRUCE, V. (in press). Face-to-face interaction and video mediated communication: A comparison of dialogue structure and co-operative task performance. Journal of Experimental Psychology: Applied.

DunCAN, S., \& FiskE, D. W. (1977). Face-to-face interaction: Research methods and theory. Hillsdale, NJ: Erlbaum.

Ellyson, S. L., Dovido, J. F., \& CoRson, R. L. (1981). Visual behaviour differences in females as a function of self-perceived expertise. Journal of Nonverbal Behaviour, 5, 164-171.

ERBER, N. P. (1974). Angle, distance and illumination on normal reception of speech. Journal of Speech \& Hearing Research, 17, 99 112

ExLINE, R. V. (1972). Visual interaction: The glance of power and preference. In J. K. Cole (Ed.), Nebraska Symposium on Motivation 1971 (pp. 163-206). Lincoln: University of Nebraska Press.

EXLINE, R. V., JONES, P., \& MACIOROWSKI, K. (1977). Race, affiliationconflict theory and mutual visual attention during conversation. Paper presented at the meeting of the American Psychological Association.

ExLINE, R. V., \& WiNTERS, L. C. (1965). Affective relations and mutual glances in dyads. In S. Tomkins \& C. Izaed (Eds.), Affect, cognition and personality (pp. 319-350). New York: Springer-Verlag.

Fisher, C., \& TokURA, H. (1995). The given-new contract in speech to infants. Journal of Memory \& Language, 34, 287-310.

FOWLER, C. (1988). Differential shortening of repeated content words produced in various communicative contexts. Language \& Speech, 28, 47-56.

FowLER, C., \& Housum, J. (1987). Talkers' signalling of 'new' and 'old' words in speech and listeners' perception and use of the distinction. Journal of Memory \& Language, 26, 489-504.

Fowler, C., \& LEVY, E. (1991). Some ways in which forms arise from functions in linguistic communications. Proceedings of the XIIth International Congress of Phonetic Sciences, 1, 279-282.

GAILEY, L. (1987). Psychological parameters of lip-reading skill. In B. Dodd \& R. Campbell (Eds.), Hearing by eye: The psychology of lip-reading (pp. 115-142). Hillsdale, NJ: Erlbaum.

GOLDMAN-EISLER, F. (1967). Sequential temporal patterns and cognitive processes in speech. Language \& Speech, 10, 122-132.

GoodwIN, C. (1981). Conversational organization: Interaction between speakers and hearers. New York: Academic Press.

Grice, H. P. (1975). Logic and conversation. In P. Cole \& J. Morgan (Eds.), Syntax and semantics: Vol. 3. Speech acts (pp. 41-58). New York: Academic Press.

Hawkins, S., \& Warren, P. (1994). Phonetic influences on the intelligibility of conversational speech. Journal of Phonetics, 22, 493 . 511.

HunNicutT, S. (1985). Intelligibility vs, redundancy-conditions of dependency. Language \& Speech, 28, 47-56.

IsAACS, E., \& CLARK, H. H. (1987). References in conversation between experts and novices. Journal of Experimental Psychology: General, 116, 26-37.

KENDON, A. (1967). Some functions of gaze direction in social interaction. Acta Psychologica, 26, 22-63.

KoOpmans-Van Beinum, F. J. (1980). Vowel contrast reduction: An acoustic and perceptual study of Dutch vowels in various speech conditions. Amsterdam: Academische Pers B. V.

Krantz, M., George, S. W., \& Hursh, K. (1983). Gaze and mutual gaze of pre-school children in conversation. Journal of Psychology, $113,9-15$.

Lieberman, P. (1963). Some effects of the semantic and grammatical context on the production and perception of speech. Language \& Speech, 6, 172-175. 
LindBlom, B. (1990). Explaining variation: A sketch of the $\mathrm{H}$ and $\mathrm{H}$ theory. In W. Hardcastle \& A. Marchal (Eds.), Speech production and speech modelling (pp. 403-439). Dordrecht: Kluwer.

MASSARO, D. W. (1989). Multiple book review of Speech perception by ear and eye: A paradigm for psychological inquiry. Behavioral \& Brain Sciences, 12, 741-794.

MASSARO, D. W., \& COHEN, M. M. (1983). Evaluation and integration of visual and auditory information in speech perception. Journal of Experimental Psychology: Human Perception \& Performance, 9, 753-771.

Mcallister, J., Potts, A., Mason, K., \& Marchant, G. (1994). Word duration in monologue and dialogue speech. Language \& Speech, 37, 393-406.

MCGuRK, H., \& MacDonald, J. W. (1976). Hearing lips and seeing voices. Nature, 264, 746-748.

Mehribian, A. (1972). Nonverbal communication. Chicago: AldineAtherton.

MehtA, G., \& CUTLER, A. (1988). Detection of target phonemes in spontaneous and read speech. Language \& Speech, 31, 135-156.

Miller, G. A., Heise, G. A., \& Lichten, W. (1951). The intelligibility of speech as a function of the context of the test materials. Journal of Experimental Psychology, 41, 338-352.

MogFoRD, K. (1987). Lip-reading in the prelingually deaf. In B. Dodd \& R. Campbell (Eds.), Hearing by eye: The psychology of lipreading (pp. 191-212). Hillsdale, NJ: Erlbaum.

Nielson, G. (1962). Studies in self-confrontation. Copenhagen: Monksgaard.

O'Malley, C., \& LANGton, S. (1994). Making the case for face-toface interaction: Empirical studies of video-mediated communication. In S. Emott \& D. Travis (Eds.), Proceedings of the International Symposium on POTS to PANS: User Issues in the Multimedia Revolution from Plain Old Telephoney Services to Pictures and Network Services. Suffolk, U.K.: British Telecom.

O'Sullivan, M., Ekman, P., Friesen, W., \& Scherer, K. (1985). What you say and how you say it: The contribution of speech content and voice quality to judgment of others. Journal of Personality \& Social Psychology, 48, 54-62.

Pollack, I., \& Pickett, J. (1963). The intelligibility of excerpts from conversation. Language \& Speech, 6, 165-171.

Prince, E. (1981). Toward a taxonomy of given-new information. In P. Cole (Ed.), Radical pragmatics (pp. 223-255). New York: Academic Press.

Reisberg, D., Mclean, J., \& Goldfield, A. (1987). Easy to hear but hard to understand: A lip-reading advantage with intact auditory stimuli. In B. Dodd \& R. Campbell (Eds.), Hearing by eye: The psychology of lip-reading (pp. 97-114). Hillsdale, NJ: Erlbaum.
SchoBer, M., \& ClaRK, H. H. (1989). Understanding by addressees and overhearers. Cognitive Psychology, 21, 211-232.

SumbY, W. H., \& Pollack, I. (1954). Visual contribution to speech intelligibility in noise. Journal of the Acoustic Society of America, 26, 212-215.

SummERFIELD, A. Q. (1987). Some preliminaries to a comprehensive account of audio-visual speech perception. In B. Dodd \& R. Campbell (Eds.), Hearing by eye: The psychology of lip-reading (pp. 352). Hillsdale, NJ: Erlbaum.

TERKEN, J., \& NoOTEBOOM, S. (1987). Opposite effects of accentuation and deaccentuation on verification latencies for Given and New information. Language \& Cognitive Processes, 2, 145-163.

Walden, B. E., Prosek, R. A., \& Worthington, D. W. (1975). Auditory and audiovisual feature transmission in hearing-impaired adults. Journal of Speech \& Hearing Research, 18, 272-280.

WALKER, M. B. (1977). The relative importance of verbal and nonverbal cues in the expression of confidence. Australian Journal of Psychology, 29, 45-57.

WALKer, M. B., \& Trimboli, C. (1983). The expressive function of the eye flash. Journal of Nonverbal Behaviour, 8, 3-13.

Wilkes-Gibbs, D., \& Clark, H. H. (1992). Coordinating beliefs in conversation. Journal of Memory \& Language, 31, 183-194.

\section{NOTES}

1. In quoted extracts, the sequence "..." indicates a short pause. Pointed brackets, $<>$, indicate a stretch of dialogue containing overlapping speech, with a slash,/, to mark the approximate point at which the second speaker interrupts the first.

2. More conservative scoring systems had little effect on the results.

3. The 47 items reintroduced in later dialogues did not differ in intelligibility effects from the remaining 24: In a $\times$ materials ANOVA on intelligibility loss with dialogue condition (face visible, face screened) within materials crossed with stimulus subgroup between them, neither the main effect of subgroup $\left[F_{2}(1,69)=1.88, p>.10\right]$ nor the interaction of subgroup with dialogue condition $\left(F_{2}<1\right)$ was significant.

4. Though movement could be seen by speakers gazing at their maps, lip shape could not. The minimum angular difference between the top of the map and the interlocutor's mouth exceeds the offset at which lip shape can be detected (Massaro, personal communication, April 1996).

(Manuscript received February 27, 1995; revision accepted for publication June 27,1996 .) 